\title{
Estudo de Colisões Inelásticas por meio da Videoanálise ${ }^{+*}$
}

Guilherme Almeida Montoli ${ }^{1}$

Graduando do curso de Licenciatura em Física

Instituto Federal de Educação, Ciência de Tecnologia do Amazonas

João dos Santos Cabral Neto ${ }^{1}$

Instituto Federal de Educação, Ciência de Tecnologia do Amazonas

Manaus - AM

\section{Resumo}

Nos vários níveis de ensino é apresentado ao estudante o fenômeno da colisão. Comumente, privilegia-se de dois tipos de colisões: perfeitamente (ou totalmente) elástica (coeficiente de restituição $C R=1$ ) e perfeitamente (ou totalmente) inelástica (coeficiente de restituição $C R$ =0). Contudo, boa parte dos fenômenos físicos em que ocorre colisão é do tipo inelástico, e por isso que perguntamos: como descrever o valor do coeficiente de restituição que assume valores $0<C R<1$ para uma colisão inelástica? Usando o método experimental em que se deixa cair uma bola ao chão, registrando o movimento da bola rebotando, pode-se determinar o valor de CR. Neste trabalho usa-se esse método experimental onde analisamos o movimento da bola por meio da videoanálise e propomos o modelo do oscilador harmônico amortecido para modelar o movimento da bola rebotando e com esse determinar CR em função da constante de amortecimento dos osciladores.

Palavras-chave: Colisão Inelástica; Coeficiente de Restituição; Tracker; Analogia.

\footnotetext{
${ }^{+}$Study of the Inelastic Collisions through Analysis Video

* Recebido: abril de 2019. Aceito: maio de 2020.

${ }^{1}$ E-mails: guilhermemontoli@gmail.com; joaocabral_68@hotmail.com
} 


\begin{abstract}
At the various educational levels is taught in the schools the phenomenon of the collision. Generally, are privileged two types of collision: perfectly elastic (restitution coefficient $C R=1$ ) and perfectly inelastic (restitution coefficient $C R=0)$. However, large part of the phenomena physic at which occurs collision are type inelastic, and that is why we are asking: how calculate the restitution coefficient taking on values $0<C R<1$ for inelastic collision? This paper, using an experiment at which we dropping a ball to the floor, we shoot the movement of the ball bouncing through analysis video find $\varepsilon$ for an inelastic collision. Furthermore, we use the damped harmonic oscillator model to build an analogy with movement of the ball and to find value of $C R$ depending of the dampening constant.
\end{abstract}

Keywords: Inelastic Collision; Coefficient of Restitution; Tracker; Analogy.

\title{
I. Introdução
}

Estudar a colisão entre partículas constitui-se num importante conteúdo no ensino de física para a compreensão de fenômenos que ocorrem na escala macroscópica, como acidente entre veículos, e na escala microscópica como as colisões entre partículas elementares observadas em aceleradores de partículas (MEIRA FILHO, 2017).

As colisões ocorrem quando duas ou mais partículas se aproximam, entram em contato e durante um curtíssimo intervalo de tempo havendo uma troca de momento e energia. Para classificar o tipo de colisão faz-se o uso do conceito de coeficiente de restituição $(\varepsilon)$, que estima a fração da perda de energia cinética antes e depois da colisão indicando o grau de elasticidade da colisão.

O primeiro contato do estudante com esse conteúdo ocorre no $1^{\circ}$ ano do ensino médio e ao chegar ao curso superior pertencente à área das Ciências da Natureza e Matemática tem geralmente seu segundo contato com a temática na disciplina de Física I. Em ambos os níveis de ensino, a abordagem teórica dada no estudo de colisões entre partículas macroscópicas privilegia os casos em que as colisões são totalmente elásticas $(\varepsilon=1)$ ou totalmente inelásticas $(\varepsilon=0)$. Mas, como demonstrar a colisão em que o coeficiente de restituição é $0<\varepsilon<1$ ?

Vários trabalhos têm sido feitos em que discutem a colisão inelástica analisando o impacto de uma bola com uma superfície rígida (LOPES, 1991; CAVALCANTE et al., 2002; AGUIAR, 2003; WADHWA, 2009; ARYAEI, 2010; CROSS, 2017). Neles o modo experimental utilizado para a demonstração de uma colisão inelástica tem sido deixar cair 
uma bola sobre uma superfície plana em repouso, analisando o sinal sonoro produzido pelo impacto ou filmando usando o recurso de câmera lenta, estimando-se o intervalo de tempo entre impactos consecutivos e calculando-se o valor do coeficiente de restituição.

Neste trabalho, implementamos o mesmo modo experimental e por meio de videoanálise estudamos o comportamento de uma bola de basquete rebotando no chão. $\mathrm{O}$ método implementado consistiu em filmar o movimento da bola colidindo com uma superfície plana, transferindo o registro do movimento para o software Tracker e analisar os dados gerados. Com os dados gerados da videoanálise determinamos o coeficiente de restituição. Considerando que o movimento da bola rebotando com a superfície plana assemelha-se ao um oscilador harmônico subamortecido, propomos o uso desse modelo como um análogo do movimento da bola para o cálculo do $\varepsilon$.

\section{Colisão inelástica e a queda livre}

Uma colisão pode resultar do contato direto (ou indireto, ambas ilustradas na figura 1) entre duas partículas e ser classificada como elástica ou inelástica a depender do valor do coeficiente de restituição $\varepsilon$, que mede a razão entre as velocidades relativas antes e depois da colisão: $\varepsilon=0$ a colisão é totalmente inelástica; $0<\varepsilon<1$ colisão inelástica; $\varepsilon=1$ colisão totalmente elástica.

Tradicionalmente na literatura são discutidos os casos limítrofes $\varepsilon=0$ e $\varepsilon=1$, estudados por meio dos princípios da conservação do momento linear e da conservação da energia. Por exemplo, considerando um movimento unidimensional em que duas bolas se movimentam sobre uma superfície e colidem, $\varepsilon=0$ significa que após a colisão as bolas permanecem em movimento, mas agora unidas e o sistema passa a ter energia cinética menor. Pode acontecer das bolas, após a colisão, permanecerem em movimento desconectadas umas das outras e também a energia cinética do sistema não ser conservada, o que implicaria $0<\varepsilon$ $<1$. Caso $\varepsilon=1$, a energia cinética do sistema é conservada, o que na realidade não acontece (nessa situação optamos por desprezar o fato de parte da energia reaparecer sob outras formas). Na prática as colisões são inelásticas porque parte da energia do sistema antes da colisão é sempre transformada em outras formas de energia (por exemplo, na produção de som e calor) durante a colisão, o que inviabiliza saber sobre as velocidades das partículas depois da colisão e, consequentemente, determinar o valor de $\varepsilon$.

A queda livre é um exemplo familiar no ensino de física que pode ser utilizado no estudo da colisão inelástica: deixa-se cair uma bola ao chão que rebotará dependendo do coeficiente de restituição ( $\varepsilon$ ) entre a bola e a superfície (Fig. 2).

Diferentes implementações deste modo experimental em que determinam o valor de $\varepsilon$ são apresentados no quadro 1 . Os valores obtidos para o coeficiente partem da medida da fração de energia cinética antes e depois da colisão supondo que essa seja constante para os $n$ impactos. Define-se a altura $h$ da queda, assumindo conhecer o valor da gravidade $g$ local, e mede-se o intervalo de tempo $\Delta t$ entre colisões sucessivas. Se assumirmos que a fração da 
energia cinética não permanece constante, $\varepsilon_{n}$ dependerá da velocidade antes e depois para cada impacto da bola com o chão.

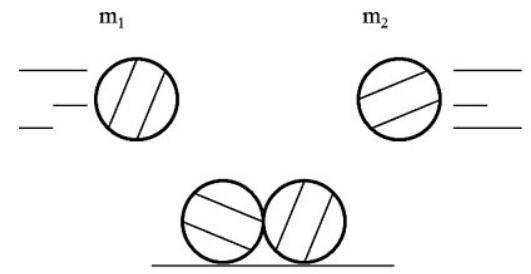

(a)

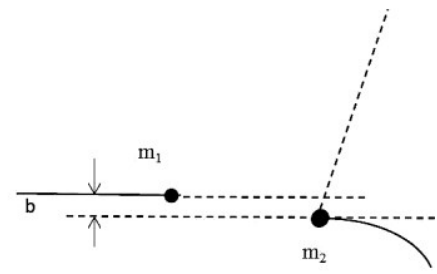

(b)

Fig. 1 - (a) Colisão direta, (b) Colisão indireta quando a força repulsiva entre as partículas produz um espalhamento.

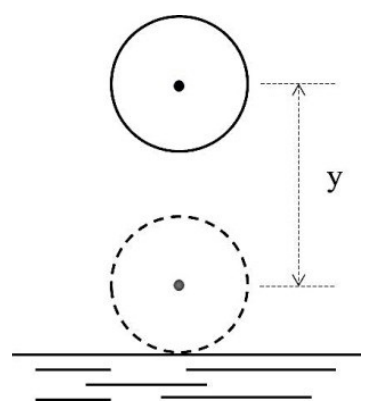

Fig. 2 -y representa a distância percorrida pelo centro de massa da bola.

Quadro 1 - Diferentes implementações para determinar o coeficiente de restituição.

\begin{tabular}{ll}
\hline \multicolumn{1}{c}{ Estudo } & \multicolumn{1}{c}{ Coeficiente de Restituição $(\varepsilon)$} \\
\hline $\begin{array}{l}\text { Se estabelece a relação } \boldsymbol{h}_{\boldsymbol{n}}=\boldsymbol{\varepsilon}^{2 n} \boldsymbol{h} \text { supondo } \\
\text { que a fração da perda de energia cinética seja } \\
\text { constante para } \boldsymbol{n} \text { impactos, em que } \boldsymbol{h} \text { é a } \\
\text { altura da queda (LOPES, 1991). }\end{array} \quad \begin{array}{c}T-\left(\frac{2 h}{g}\right)^{\frac{1}{2}} \\
T+\left(\frac{2 h}{g}\right)^{\frac{1}{2}}\end{array}, T$ é o tempo que a bola permanece \\
pulando.
\end{tabular}


(No que tange ao que é discutido neste trabalho.) A partir da filmagem dos quiques da bola, e utilizando o recurso da câmera lenta, foi estabelecida a relação entre o coeficiente de restituição e as alturas alcançadas pela bola entre dois impactos $\varepsilon=\sqrt{\frac{h_{n+1}}{h_{n}}}$, em que $h_{n}$ é a altura alcançada após o consecutivos (ARYAEI, 2010).

Fonte: Elaborado pelos autores.

A bola deixada cair da altura $\mathrm{h}$ rebotará várias vezes até parar, consumindo um tempo total

$$
T=t_{1}+2 \sum_{k=2}^{n-1} t_{k}
$$

onde $t_{k}=\sqrt{\frac{2 y_{k}}{g}}, y_{k}$ é a altura da queda e $t_{1}=\sqrt{\frac{2 h}{g}}$. O módulo da velocidade da bola ao atingir o chão será $v_{n}=\sqrt{2 g y_{n}}$, que para $n=1, v_{1}=\sqrt{2 g h}$. A fração da perda de energia cinética devido ao impacto com o chão é definida como

$$
f_{n}=\frac{K_{n}-K_{n+1}}{K_{n}}=1-\frac{v_{n+1}^{2}}{v_{n}^{2}} .
$$

$\mathrm{O}$ valor do coeficiente de restituição para o $n$-ésimo impacto é definido como $\varepsilon_{n}=\frac{v_{n+1}}{v_{n}}$ (LOPES, 1991; CAVALCANTE et al., 2002), assim temos $f_{n}=1-\varepsilon_{n}^{2}$. No caso da colisão totalmente elástica da bola com o chão $f=0$ e totalmente inelástica em que toda a energia da bola é dissipada no impacto $f=1$. Usando essa definição, encontramos

$$
v_{n+1}=v_{1} \prod_{k=1}^{n} \varepsilon_{k} .
$$

e o tempo total que a bola permaneceu pulando, usando o valor para $t_{1}$ e a relação $y_{n+1}=$ $y_{n} \varepsilon_{n}^{2}$, ficamos com

$$
T=\sqrt{\frac{2 h}{g}}\left[1+2 \sum_{k=1}^{n-1}\left(\prod_{j=1}^{k} \varepsilon_{j}\right)\right] .
$$

Considerando que a perda da fração da energia cinética é constante (LOPES, 1991; AGUIAR, 2003; ARYAEI, 2010) as eq. (3) e (4) reduzem-se a

$$
\begin{gathered}
v_{n+1}=\varepsilon^{n} v_{1}, \\
T=\sqrt{\frac{2 h}{g}}\left[1+2 \sum_{k=1}^{n-1} \varepsilon^{k}\right] .
\end{gathered}
$$


A eq.(6) quando expandida assume a forma de uma progressão geométrica cuja razão é $\varepsilon$. O tempo total nessa condição para n pulos ficará escrita por

$$
T=\sqrt{\frac{2 h}{g}}\left[\frac{1+\varepsilon\left(1-2 \varepsilon^{n-2}\right)}{(1-\varepsilon)}\right] .
$$

Lopes (1991) utilizou desse recurso matemático e encontrou o valor do coeficiente de restituição que é apresentado no quadro 1. Por outro lado, pode-se determinar o tempo de voo entre dois impactos consecutivos $t_{n}=2 \sqrt{\frac{2 y_{n}}{g}}$, de acordo com a eq.(1), e relacionar com o valor da velocidade $v_{n}=\sqrt{2 g y_{n}}$, resultando em

$$
\log \left(t_{n}\right)=\log \left(t_{1}\right)+n \log (\varepsilon)
$$

A partir do o gráfico $\log \left(t_{n}\right) \times n$ [4], foi determinada a expressão matemática do valor de $\varepsilon$, mostrada no quadro 1 .

Usando o mesmo procedimento experimental, coletamos os dados que possibilitam calcular o valor do coeficiente de restituição por meio do recurso tecnológico da videoanálise com o software Tracker. Comparamos os valores encontrados do coeficiente de restituição usando o Tracker com os apresentados no quadro 1, que são mostrados no quadro 2.

\section{Analogia com o oscilador amortecido}

O estudo do oscilador harmônico simples apresenta uma ampla variedade de aplicações pedagógicas no ensino de física como na resolução de problemas reais, em que a existência de algum tipo de mecanismo produz perda de energia é considerado como um amortecimento no movimento de oscilação. A bola quando colide com o chão e rebota várias vezes desperta o nosso olhar para pensar nesse movimento como um sistema análogo a um sistema massa-mola amortecido sujeito a uma força de atrito $f=-m \gamma \dot{y}$, em que $\gamma$ é a constante de amortecimento. A variação da altura da bola após cada impacto apresenta uma similaridade com $|y(t)|$ da solução $y(t)$ do oscilador harmônico subamortecido porque reproduz qualitativamente o comportamento da bola rebotando do chão. A bola desloca-se como se estivesse ligada a um sistema elástico hipotético de constante elástica $k$ em paralelo a um amortecedor de constante de amortecimento $\gamma$. Consideramos que na colisão da bola com o chão o processo de deformação da bola não ultrapassa seu limite elástico dando-se a transformação de energia cinética em energia potencial e vice-versa com dissipação de energia. De acordo com texto básico de mecânica clássica (THORNTON; MARION, 2011), a equação de movimento de um oscilador harmônico amortecido para o regime de subamortecimento é dada por:

$$
y(t)=h e^{-\frac{\gamma}{2 m} t}\left[\cos (w t)+\frac{\gamma}{2 m w} \operatorname{sen}(w t)\right],
$$


de modo a atender as condições iniciais: $y(0)=h$ e $\dot{y}(0)=0$, onde $h$ é a altura inicial da queda medida do chão, $g$ o valor da gravidade, $m$ a massa da bola, $k$ a constante elástica da mola hipotética e $w=\sqrt{\frac{k}{m}-\frac{\gamma^{2}}{4 m^{2}}}$ a frequência com que a bola colide com o chão e rebota.

Nesta modelagem a eq. (2), que define fração da perda de energia cinética devido ao impacto com o chão, é determinada calculando-se a energia cinética do hipotético oscilador harmônico subamortecido entre dois impactos consecutivos, ou seja,

$$
f_{n}=1-\frac{K\left(t+\theta_{n}\right)}{K(t)}=1-e^{-\frac{\gamma}{m} \theta_{n}}
$$

onde $\theta_{n}=\frac{2 \pi}{w}$ é o tempo entre o n-ésimo impacto e o $\mathrm{n}+1$-ésimo impacto. Comparando eq.(10) com eq.(2), encontramos o coeficiente de restituição

$$
\varepsilon_{n}=e^{-\frac{\gamma}{2 m} \theta_{n}} .
$$

Portanto, o valor do coeficiente de restituição calculado por meio do modelo do oscilador subamortecido depende da constante de amortecimento $\gamma$ e do tempo entre impactos consecutivos. No caso em estudo adotamos um valor para $\gamma$ que simula o movimento da bola rebotando e $\theta_{n}$ o tempo entre o primeiro e segundo impactos.

\section{O Experimento}

O procedimento adotado na análise da colisão consiste na organização do experimento com a definição do cenário para a filmagem, na filmagem do movimento da bola colidindo com a superfície até parar de rebotar, na transferência do vídeo para o computador e na observação dos dados gerados pelo software Tracker.

No experimento utiliza-se um smartphone com a função filmadora, um tripé para apoiar o smartphone, uma bola de basquete e um objeto servindo de elemento para a calibragem da escala do vídeo. Usamos a bola de basquete devido ao seu tamanho, o que ajuda na qualidade do vídeo, além de ser é um objeto produzido para rebotar ao colidir com o chão.

\section{a. Filmagem}

No processo de filmagem é importante observarmos o correto posicionamento da câmera e a qualidade do vídeo. Apoiamos o smartphone (estando no modo avião para evitar interrupções) num tripé para evitar tremulações no vídeo, posicionado horizontalmente e nivelado. A distância entre a câmera e o objeto que estava sendo filmado (a bola de basquete) foi ajustada para que o enquadramento do filme registrasse o movimento de queda da bola ao chão até parar de rebotar. Foram produzidos vídeos com qualidade (ou definição) full hd (1920x1080 pixels) e com resolução de 30 e 60 qps (quadros por segundo). É necessário lembrar que, na captação do movimento da bola caindo, colidindo com o chão e rebotando, a 
resolução da câmera é um fator importante para evitar que a imagem da bola fique borrada (exibam rastro) dificultando a análise de dados. Portanto, antes de iniciar a filmagem, é necessário observar na configuração do smartphone a resolução da câmera. Para uma câmera com resolução de 30 qps teremos aproximadamente a cada $0,033 \mathrm{~s}$, o registro de um quadro em que aparece a bola em queda ou rebotando. Quando filmamos com 60 qps, registra-se aproximadamente um quadro a cada $0,017 \mathrm{~s}$. Justapondo quadro a quadro registrado pela câmera compõe-se o filme da queda da bola e os respectivos rebotes. Por exemplo, considere que a altura de queda livre (sem resistência do ar) de uma bola seja $h=1,00 \mathrm{~m}$ e que a aceleração da gravidade local seja $\mathrm{g}=9,80 \frac{\mathrm{m}}{\mathrm{s}^{2}}$; a bola levará aproximadamente $0,45 \mathrm{~s}$ para chegar ao chão, o que corresponderia aproximadamente a 14 quadros registrados pela câmera e transformados em filme. Se a quantidade de quadros por segundo for 60 , teríamos aproximadamente 27 quadros ao final da queda, dobrando a resolução temporal. Informações e ajustes na resolução do filme pode ser visto no Tracker na barra do menu comando clip settings (configurações dos quadros).

\section{b. Transferência do vídeo}

Transferido o vídeo para uma pasta no computador no formato .mp4, importamos o vídeo para dentro do software Tracker. Para que se inicie a análise do vídeo e a consequente geração de dados é necessário calibrar o vídeo no software.

\section{c. Dados gerados}

O Tracker possui uma biblioteca contendo várias variáveis de interesse no estudo da dinâmica do movimento. Se a variável de interesse não constar na biblioteca, o software permite a inserção de nova variável a qual é definida a partir das variáveis já existentes na biblioteca. Para que os dados de interesse sejam gerados é necessário calibrar o vídeo no Tracker. O processo de calibragem propiciará que a análise de vídeo feita pelo software gere dados reais e se faz com três procedimentos: (1) usar o recurso contido no menu principal do software chamado Fita Métrica com Transferidor informa-se o tamanho real de um objeto que faz parte do cenário do vídeo. O software fará a videoanálise tomando como escala o tamanho do objeto; (2) escolher um ponto no cenário do vídeo com a origem do referencial usando-se o recurso Eixos de Coordenadas do menu principal; (3) definir a localização da origem do sistema de referência, sendo necessário marcar quadro a quadro o ponto que indicará o movimento do objeto de interesse usando o recurso Ponto de Massa no menu principal. Isso é feito mantendo-se a tecla shift acionada e clicando com o botão do lado esquerdo do mouse sobre a bola; o Tracker passará quadro a quadro o vídeo para que seja marcado o ponto na bola (utilizamos um vídeo com 60 qps resultando em 488 quadros de aproximadamente 8,15 segundos). Neste estudo definimos como ponto de massa para análise e geração de dados o centro geométrico da bola. 
Ao tratarmos o movimento de queda da bola por meio da videoanálise podemos ter a impressão que os valores das grandezas físicas de interesse têm valor absoluto. Sabemos que na realização de um experimento vários fatores podem produzir erros na medida de uma grandeza física. No caso em que trabalhamos os valores das grandezas foram gerados por medida indireta (gerados pelo software Tracker) e para minimizar possíveis erros deve-se observar o posicionamento da câmera, a qualidade do vídeo e a repetição do experimento para escolher o vídeo que será analisado. Por mais cuidadoso que o experimentador seja estamos susceptíveis a erros.

A metodologia utilizada para estimar os erros associados aos valores gerados da videoanálise considera o fato de que a bola na altura máxima está praticamente em repouso ou muito próximo disso:

(i) Incerteza na medida do tempo: corresponde ao intervalo de tempo entre quadros ficando o valor $0,02 \mathrm{~s}$.

(ii) Incerteza na medida da altura: foi estimada comparando os valores da altura máxima atingida pela bola extraídos da videoanálise com o do modelo teórico para o oscilador harmônico subamortecido, e para isso utilizamos o termo modulador das oscilações $h e^{-\frac{\gamma}{2 m} t}$, ajustando aos dados experimentais pelo método dos mínimos quadrados resultando no valor $0,04 \mathrm{~m}$ (coeficiente de correlação $R=-0,99861$ ).

(iii) $\mathrm{O}$ valor adotado para a aceleração da gravidade local foi $(9,8 \pm 0,1) \mathrm{m} / \mathrm{s}^{2}$.

$O$ valor do coeficiente de restituição $\varepsilon$ é obtido por meio de medida indireta usando gráficos e expressões matemáticas cuja incerteza irá depender das incertezas das grandezas física envolvidas. A incerteza no valor de $\varepsilon$ resulta da propagação dessas incertezas. $O$ valor da incerteza de $\varepsilon$ a partir das expressões matemáticas (quadro 1) depende da incerteza na medida de h (altura), T (tempo) e g (gravidade), que foram efetuadas usando a videoanálise, isto é, sem uso de instrumento de medida, cujas incertezas são mencionadas anteriormente. Os valores de $\mathrm{h}$ e $\mathrm{T}$ foram obtidos da videoanálise do vídeo escolhido (aquele com melhor qualidade), e esses foram usados para determinar a incerteza que envolve expressões matemáticas no cálculo de $\varepsilon$ por meio da fórmula de propagação de incertezas: $\sigma_{\varepsilon}^{2}=$ $\left(\frac{\partial f}{\partial h}\right)^{2} \sigma_{h}{ }^{2}+\left(\frac{\partial f}{\partial T}\right)^{2}{\sigma_{T}}^{2}+\left(\frac{\partial f}{\partial g}\right)^{2} \sigma_{g}{ }^{2}$, em que $\sigma_{\varepsilon}^{2}$ é a variância da função $\varepsilon=f(h, T, g)$.

Para a determinação da incerteza do valor de $\varepsilon$, quando determinado por meio de gráfico, usamos o método dos mínimos quadrados por meio de recurso computacional.

\section{O valor do coeficiente de restituição}

O movimento de queda da bola foi filmado utilizando um smartphone e o vídeo transferido para o software Tracker e, após o processo de calibragem, iniciou-se a videoanálise (na Fig. 3 apresentamos uma imagem do vídeo). Uma folha de papel de $41 \mathrm{~cm}$ de comprimento foi utilizada para a calibração do vídeo. 


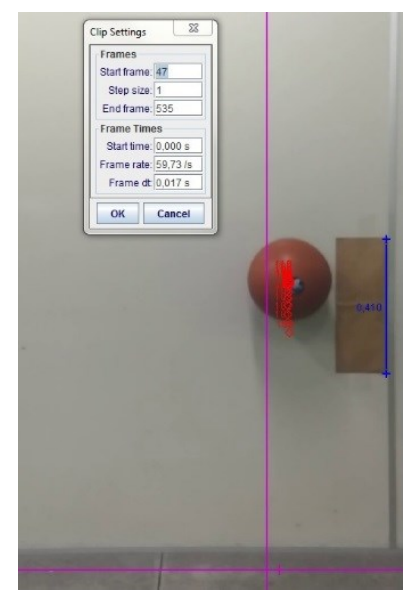

Fig. 3- Imagem da bola em queda livre capturada pela câmera do smartphone.

Os resultados da videoanálise são apresentados nas janelas de Dados e Gráficos cujos valores de interesse foram: o tempo total $\mathrm{T}$ que a bola permanece rebotando até parar, o comportamento da altura alcançada pelo centro de massa da bola $\mathrm{y}(\mathrm{t})$, os intervalos de tempo $\Delta \mathrm{t}$ entre dois impactos consecutivos, os comportamentos da velocidade vertical $v(t)$ e da aceleração vertical $a(t)$. Com os dados gerados pela videoanálise construímos o quadro 2 , onde apresentamos o valor do coeficiente de restituição $\varepsilon$ resultante da colisão da bola com o chão, e comparamos com os estudos indicados no quadro 1. A bola de basquete rebotou 25 vezes até parar consumindo, aproximadamente, um tempo total $\mathrm{T}=8,15 \pm 0,02 \mathrm{~s}$.

Quadro 2 - Valores do coeficiente de restituição a partir de dados gerados por meio da videoanálise e aplicados nas perspectivas dos estudos apresentados no quadro1 $(n=25$ impactos).

\begin{tabular}{|c|c|c|}
\hline Estudo & $\begin{array}{l}\text { Coeficiente de } \\
\text { Restituição }(\varepsilon)\end{array}$ & Detalhamento do Cálculo \\
\hline Lopes (1991) & $0,868 \pm 0,002$ & $\begin{array}{l}\text { Expandimos a eq.(6) para } \mathrm{n}=25 \text { impactos e por meio } \\
\text { de recurso computacional encontramos o valor de } \varepsilon \text {. }\end{array}$ \\
\hline Cavalcante et al (2002) & $0,85 \pm 0,04$ & $\begin{array}{l}\text { Construímos o gráfico } \Delta t_{n+1} \times \Delta t_{n} \text { mostrado na figura } \\
2 \text { (a) e } \varepsilon \text { é calculado pela inclinação da reta. }\end{array}$ \\
\hline Aguiar (2010) & $0,93 \pm 0,03$ & $\begin{array}{l}\text { Extraindo da videoanálise o tempo } t_{n} \text { de voo entre o } n \text { - } \\
\text { ésimo e o }(n+1) \text {-ésimo impacto. A relação entre os } \\
\text { tempos e o número de impacto n é mostrada na figura } \\
\text { 2(b). }\end{array}$ \\
\hline Wadhwa (2009) & $0,94 \pm 0,03$ & $\begin{array}{l}\text { Escolhemos o intervalo de tempo entre o primeiro e } \\
\text { segundo impactos gerados pela videoanálise e } \\
\text { aplicamos na relação proposta. }\end{array}$ \\
\hline Aryaei (2010) & $0,87 \pm 0,03$ & $\begin{array}{l}\text { A videoanálise produz o comportamento da altura } \\
\text { alcançada após cada impacto. A relação entre as } \\
\text { alturas, mostrada na figura } 2 \text { (c), permite encontrar o } \\
\text { valor do coeficiente } \varepsilon \text {. }\end{array}$ \\
\hline
\end{tabular}

Fonte: Elaborado pelos autores. 
Na Fig. 4 mostramos os gráficos os quais, que por meio da inclinação da reta, permitiu-nos encontramos o valor de $\varepsilon$ segundo os estudos apresentados no quadro 1 . No quadro 2, temos esses valores com suas respectivas incertezas.

O valor calculado do coeficiente de restituição por meio da modelagem do comportamento da altura alcançada, após cada impacto da bola com o chão, com o oscilador harmônico subamortecido, é estimado para o primeiro ciclo de oscilação. Deste modo, $\theta_{n}$ da eq. (11), é determinado na videoanálise como o valor do tempo entre o primeiro e segundo impactos cujo valor é $\theta_{n}=(0,92 \pm 0,02)$ s. O valor adotado para constante de amortecimento $\gamma$ que simula o comportamento qualitativamente da bola rebotando em acordo com a eq.(11) é $0,39 \mathrm{Kg} / \mathrm{s}$. A massa da bola de basquete é $m=(0,62 \pm 0,05) \mathrm{Kg}$. A substituição desses valores na eq. (11), resulta em $\varepsilon=(0,75 \pm 0,02)$.
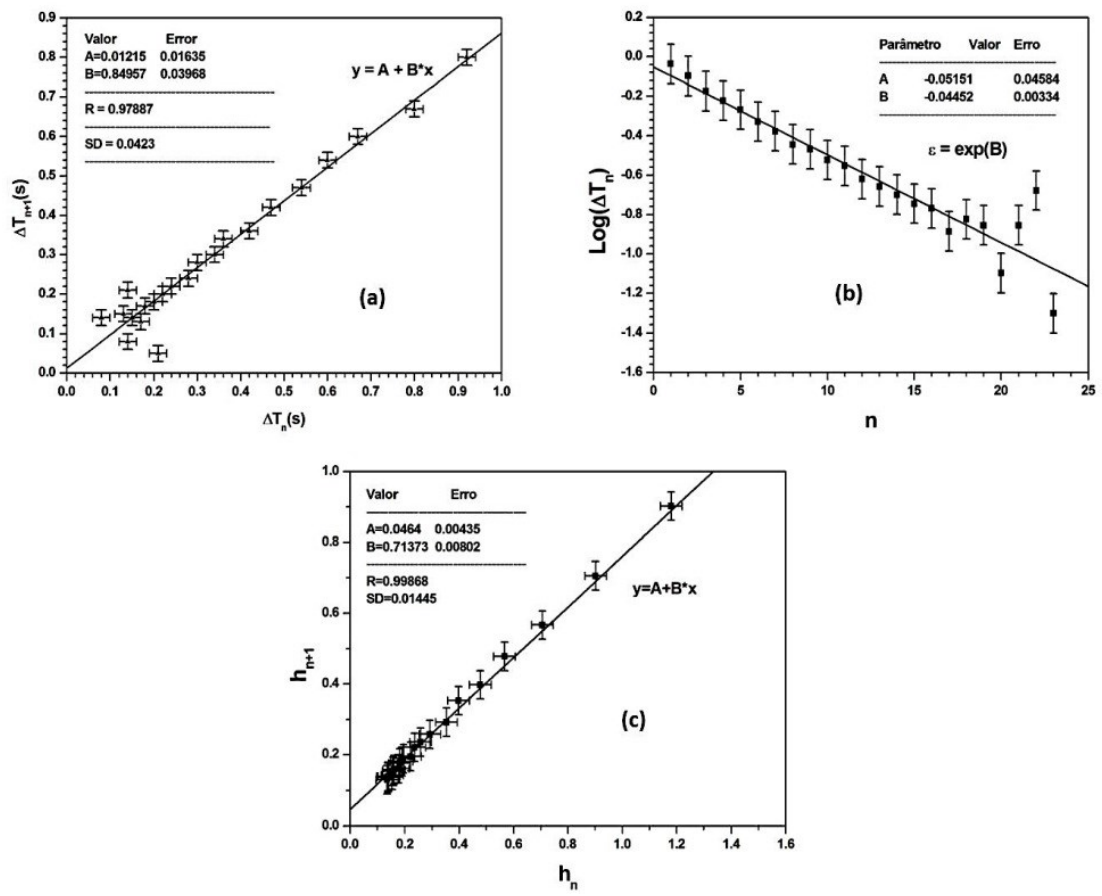

Fig. 1 - (a) Relação entre intervalos de tempo consecutivos entre impactos $\Delta t_{(n+1)} \times \Delta t_{n}$, (b) Relação entre o tempo de voo $t_{n}$ entre o n-ésimo e o $(n+1)$-ésimo impacto versus $n$ e (c) Relação entre as alturas alcançadas entre impactos consecutivos.

O comportamento do coeficiente de restituição em função da constante de amortecimento do nosso modelo pode ser visto na Fig. 5, onde mostramos $\varepsilon$ para valores de $\gamma$ cujo regime oscilatório é subamortecido. Neste modelo, se fizermos $\gamma \geq 3$, o sistema assume o regime amortecido crítico, que no experimento corresponderia a bola ao ser deixada cair ao chão não rebotar. 


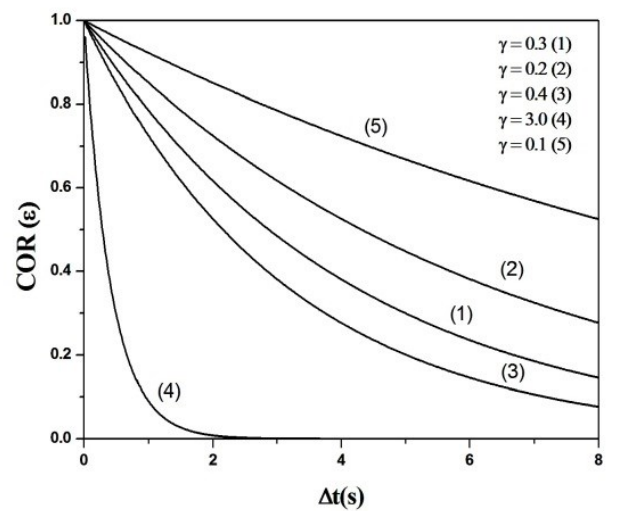

Fig. 2 - Comportamento do \& para vários valores da constante de amortecimento.

\section{Impulso da Força}

A força de contato entre a bola e o chão que atua durante a colisão num curto intervalo de tempo também pode ser estimada por meio da videoanálise. A força impulsiva para cada impacto da bola com o chão é mostrada na figura 6 em que a área hachurada, debaixo da curva $\mathrm{F}(\mathrm{t})$, representa o impulso da força.

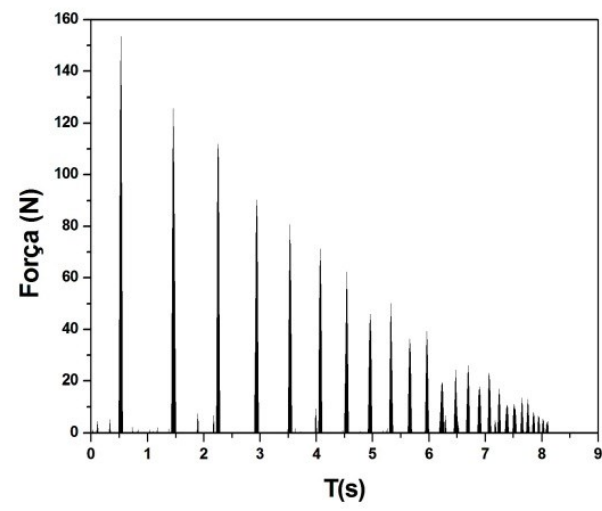

Fig. 6 - Força impulsiva sobre a bola.

No processo da videoanálise os valores gerados, em que são calculados o valor da aceleração experimentada pela bola a partir da justaposição de quadros do movimento de queda, devem considerar a força da gravidade e a força de atrito que impede a bola de deslizar no chão.

\section{Considerações finais}

Neste trabalho, estudamos a colisão inelástica no limite onde o coeficiente de restituição está no intervalo $0<\varepsilon<1$ por meio da videoanálise usando o software Tracker. $\mathrm{O}$ 
sistema no qual observamos a colisão consistiu de uma bola em queda livre rebotando no chão até parar. Registramos o vídeo do movimento da bola e os dados gerados na videoanálise possibilitaram encontrar o valor do coeficiente de restituição utilizando as várias metodologias desenvolvidas nos estudos apresentados no quadro 1. Nesses estudos considerase que a taxa de perda da energia cinética seja constante e, portanto, $\varepsilon$ é constante. Mas os resultados mostraram que o coeficiente não se mantém constante variando a cada impacto da bola com o chão. Os resultados encontrados para $\varepsilon$ são próximos, dentro da barra de erro.

Construímos uma analogia do movimento da bola com o oscilador harmônico subamortecido e encontramos uma expressão para o coeficiente de restituição que depende da constante de amortecimento do modelo. A constante de amortecimento $\gamma$ no modelo afeta $o$ coeficiente de restituição de acordo com a eq.(12). A força impulsiva que atua durante o impacto da bola com o chão pode ser estimada para cada impacto da bola com o chão.

Portanto, utilizando o software Tracker como recurso tecnológico para analisar o movimento de uma bola colidindo com o chão pode-se estudar e apresentar um modo para se discutir a colisão inelástica no ensino de física, um procedimento possível de ser implementado em sala de aula, em que é permitindo acompanhar e coletar dados em tempo real.

\section{Agradecimentos}

Agradecemos a FAPEAM pelo apoio dado no desenvolvimento do trabalho que é decorrente do programa da iniciação científica fomentado por esta agência. Agradecemos ao prof. Dr. José Ricardo de Sousa pelas edificantes discussões sobre o trabalho.

\section{Referências bibliográficas}

AGUIAR, C. E.; LAUDARES, F. Listening to the coefficient of restitution and the gravitational acceleration of a bouncing ball. American Journal of Physics, v. 71, n. 5, p. 499-501, 2003.

ARYAEI, A.; HASHEMNIA, K.; JAFARPUR, K. Experimental and numerical study of ball size effect on restitution coefficient in low velocity impacts. International Journal of Impact Engineering, v. 37, n. 10, p. 1037-1044, 2010.

CAVAlCANTE, M. A. et al. O estudo de colisões através do som. Revista Brasileira de Ensino de Física, v. 24, n. 2, p. 150-157, 2002.

CROSS, R. Impact of a ping-pong ball. Physics Education, v. 52, n. 3, p. 1-3, 2017. 
LOPES, W. Determinação do coeficiente de restauração entre uma bola e o piso do laboratório. Caderno Catarinense de Ensino de Física, v. 8, n. 3, p. 237-240, 1991.

MEIRA FILHO, D. P.; KAMASSURY, J. K. S.; MEIRA, R. C. S. Uma discussão sobre o coeficiente de restituição. Revista Brasileira de Ensino de Física, v. 39, n. 4, p. 1-11, 2017.

THORNTON, S. T.; MARION, J. B. Dinâmica clássica de partículas e sistemas. 5. ed. São Paulo: CENGAGE, 2011. 608 p.

WADHWA, A. Measuring the coefficient of restitution using a digital oscilloscope. Physics Education, v. 44, n. 5, p. 517-512, 2009. 